\title{
Anterolateral alopecia of the leg. Brief research
}

\section{on men}

\section{Piotr Brzezinski ${ }^{1,2}$, Lorenco Martini ${ }^{3}$}

${ }^{1}$ Institute of Biology and Environmental Protection, Pomeranian Academy, Slupsk, Poland, ${ }^{2}$ Department of Dermatology, Provincial Specialist Hospital in Slupsk, Ustka, Poland, ${ }^{3}$ University of Siena, Department of Pharmaceutical Biotechnologies, Via A. Moro 2, 53100 Siena, Italy

Corresponding author: Dr. Piotr Brzezinski, E-mail: brzezoo77@yahoo.com

\begin{abstract}
Background: Anterolateral leg alopecia also called peroneal alopecia is a common form of hair loss seen in adults in clinical practice but less commonly described in literature. Methods and Materials: About 903 men were included in this study. Each patient was examined for hair loss on the lower legs. A full history was taken from each patient including: age, and the presence of the analyzed skin lesions. Patients with anterolateral alopecia of the leg were asked about the treatment of this disease, co-morbidities and medications. Results: Out of the total number of 903 participants ages ranged from 20 to 52 years with a mean 36. Anterolateral leg alopecia was diagnosed by 39,97\% in patient of age from 36-52. Conclusion: The incidence of acquired frictional alopecia of the lower extremities may be greater than reflected in previously published reports.
\end{abstract}

Key words: Anterolateral leg alopecia; Hair loss; Nonscarring alopecia

\section{INTRODUCTION}

Anterolateral leg alopecia, a common form of hair loss, usually occurs in middle-aged and elderly men but also occurs in women $[1,2]$. This peculiar form of alopecia is described in few contemporary dermatology textbooks; where it is described, details are scarce. Anterolateral alopecia presents as bilaterally symmetrical, sharply demarcated, circumscribed, smooth patches of alopecia confined to the anterior and lateral aspects of the leg.2 Occasionally, a few hairs grow within the patches. Broken hairs are not common $[2,3]$.

\section{METHODS AND MATERIALS}

This study was carried in Poland. About 903 men were included in this study. Each patient was examined for hair loss on the lower legs. A full history was taken from each patient including: age, and the presence of the analyzed skin lesions. Patients with anterolateral alopecia of the leg were asked about the treatment of this disease, co-morbidities and medications. The study was not carried out in dermatological offices. Part two of patients were candidates, volunteers for military service - national reserve forces (722 participants; $79,96 \%$ - Group I), the second part reported on the study of melanocytic nevus (Melanoma Day) (181 participants; 20,04\%-Group II).

\section{Inclusion Criteria}

Age above 20 years (only men).

Discovered as an incidental finding on cutaneous physical examination during their office visit for an unrelated dermatologic complaint.

\section{Exclusion Criteria}

Age less than 20 years. Women. Participants not willing for the necessary investigations. 


\section{Ethics}

This study was performed on human subjects; thus, all patients were aware of the presence of the study and they were fully informed about this study.

\section{Statistics}

Non-normal distributions were verified with a ShapiroWilk test. U-Mann-Whitney and Kruskal-Wallis tests were used to compare the differences between the two groups. $\mathrm{P}<0.05$ was considered statistically significant. All data analysed by STATISTICA 10.

\section{RESULTS}

Out of the total number of 903 participants ages ranged from 20 to 52 years with a mean 36 (Standard Deviation, $S D \pm 3.49$ ). Anterolateral leg alopecia was diagnosed by 361 participants, 39,97\% in patient of age from 36-58. Diseases that occurred in people diagnosed with APL. Skin lesions lasted from 1 to 8 years with a mean 4,5 (SD \pm 1.19 years).

353 of participants $(97,78 \%)$ did not realize the existence of such skin lesions. The current skin lesions did not bother them, or they did not know that they exist. 39 participants $(10,04 \%)$ they had other diseases. 31 persons $(79,49 \%)$ complained of skin problems and 9 persons $(23,07 \%)$ had other systemic illnesses. Of skin diseases acne/folliculitis (22 participants), seborrheic dermatitis (9 participants). Of systemic diseases arterial hypertension (4 participants), spine joint diseases (3 participants), gout (1 person), prostate hypertrophy (1 person).

\section{DISCUSSION}

The etiology of anterolateral leg alopecia has remained largely a mystery. Kidd described anterolateral leg alopecia as resulting from "use inheritance" (patients with this condition wore tight-fitting ancestral generation shoes). Some have theorized that leg crossing contributes to the condition [4], whereas others have pointed to friction and trouser rubbing [5]. Adams attributed a young male patient's case of "waterslide alopecia" of the lower extremities to friction occurring during recreational water-slide activity [6]. Other investigators, however, have refuted proposed connections with trauma and friction. Tommasi suggested a possible relation between this pattern of alopecia and an endogenous "neuroarthritic diathesis" and hyperuricemia, but evidence of such a connection was limited [7].

Robertson believed that anterolateral leg alopecia is related to androgenetic alopecia and preferred the term patterned alopecia [8]. He suggested a disturbance in "male sexual secretion" as the etiology for the leg alopecia.

Although both forms of alopecia can often present together, Hamilton showed no statistically significant association between male pattern baldness and alopecia of the lower extremities [9].

Popkin suggested acute ischemia as a contributing factor in hair loss involving the lower extremities [10].

Kligman remarked that, aside from loss of fine hairs on the dorsum of the toes late in severe atherosclerosis obliterans, growth of leg hair is not correlated with any kind of atherosclerotic disease [11].

In short, various hypotheses have been put forward to explain anterolateral leg alopecia, but none has been substantiated, and no causal factor has been identified.

Most male patients say, as in our study that they never noticed the onset of loss of leg hair or never paid serious attention to it. The course of this condition is benign, progression is rare, and spontaneous resolution occasionally occurs.

Clinically, the main differential diagnosis is alopecia areata. A treatment outline for this common disorder also is not described in the literature.

\section{CONCLUSION}

The incidence of acquired frictional alopecia of the lower extremities may be greater than reflected in previously published reports.

\section{Statement of Human and Animal Rights}

All procedures followed were in accordance with the ethical standards of the responsible committee on human experimentation (institutional and national) and with the Helsinki Declaration of 1975, as revised in 2008 .

\section{Statement of Informed Consent}

Informed consent was obtained from all patients for being included in the study. 
www.odermatol.com

\section{REFERENSES}

1. Gupta SN, Shaw JC. Anterolateral leg alopecia revisited. Cutis. 2002;70:215-6.

2. Shetty VM, Pai SB, Pai K, Jenson JJ. Anterolateral leg alopecia: unknown entity or yet underreported? Int J Dermatol. 2018 Sep 28. doi: $10.1111 /$ ijd.14260.

3. Srinivas SM, Sacchidanand S, Jagannathan B. Anterolateral Leg Alopecia. Int J Trichology. 2016;8:49-50.

4. Siah TW, Harries MJ. Anterolateral leg alopecia: common but commonly ignored. Int J Trichology. 2014;6:75-6.

5. Turpie ID. Localized hair loss in an elderly man. CMAJ. 1983;129:537.

6. Adams BB. Water-slide alopecia. Cutis. 2001;67:399-400.

7. Tommasi L. Alopecia of the peroneal regions as a constitutional sign of neuro-arthritic diathesis. Br J Dermatol. 1940;52:1-9.
8. Robertson PC. Description and study of an area of atrophic skin occurring in men, with its relationship to the common type of diffuse alopecia of the scalp. Br J Dermatol Syph. 1938;50:581.

9. Hamilton JB. Patterned loss of hair in man: types and incidence. Ann N Y Acad Sci. 1951;53:708-728.

10. Popkin RJ. Relation of leg hair loss to arteriosclerosis. JAMA. 1970;213:130.

11. Kligman AM. Is there a relation between leg hair loss and arteriosclerosis? JAMA. 1970;212:328.

Copyright by Piotr Brzezinski, et al. This is an open-access article distributed under the terms of the Creative Commons Attribution License, which permits unrestricted use, distribution, and reproduction in any medium, provided the original author and source are credited.

Source of Support: Nil, Conflict of Interest: None declared. 